\title{
Condiçóes de saúde de idosos institucionalizados: contribuiçóes para ação interdisciplinar e promotora de saúde
}

\author{
Rosane Seeger da Silva ${ }^{a}$ (D), Elenir Fedosse ${ }^{b}$ (D), Fernanda dos Santos Pascotini ${ }^{a}$ (D), \\ Estefânia Brunelli Riehs ${ }^{\mathrm{c}}$
}

\author{
${ }^{a}$ Universidade Federal de Santa Maria - UFSM, Santa Maria, RS, Brasil.

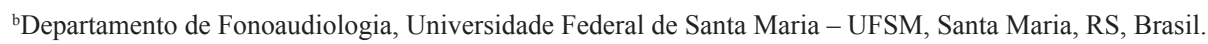 \\ 'Associação Santamariense de Auxílio aos Necessitados, Santa Maria, RS, Brasil.
}

\begin{abstract}
Resumo: Introdução: A avaliação do estado de saúde dos idosos institucionalizados é considerada uma prática essencial à promoção e recuperação da saúde, que deve incluir a investigação de aspectos relacionados às necessidades biopsicossociais. Objetivo: Caracterizar clinicamente os idosos residentes em uma ILPI filantrópica de uma cidade do interior do RS, visando o planejamento de ações interdisciplinares que preservem a independência e/ou previnam dependência funcional desses idosos. Método: Estudo transversal, descritivo e quantitativo, fundamentado na análise de um documento denominado Ficha de Cadastro que compõe o prontuário dos idosos. Foram analisadas a totalidade dos cadastros, entre os meses de maio e junho de 2017. As informações pesquisadas foram: sexo, idade, etnia/raça, escolarização, profissão/ocupação prévia, estado civil, presença de doenças e uso de medicações, bem como manutenção ou não de vínculo familiar. Resultados: Participaram da pesquisa 67 idosos, todos do sexo masculino. A idade variou entre 60 e 105 anos, sendo que a maioria se encontrava na faixa etária dos 70 anos ou mais. Identificou-se a predominância de idosos brancos, alfabetizados, profissionais de serviços gerais e solteiros; foram mais comuns os déficits visuais (maioria corrigida) e os auditivos (minoria corrigida). Destacaram-se as condições de saúde relacionadas à história de uso abusivo de álcool e tabaco, de doenças sistêmicas e neurodegenerativas, seus agravos e consequências desfavoráveis, bem como uso excessivo de medicamentos psicotrópicos e bom nível de manutenção de vínculo familiar. Conclusão: Constataram-se situações de ordem biológica e psicossocial, abordadas pela atual equipe, entre outras que requerem a atuação de novos profissionais de saúde que não compõem a equipe. Esses dados podem auxiliar no planejamento de ações voltadas à promoção de saúde, incluindo atenção interdisciplinar - ampliada, individualizada e coletiva - ao idoso institucionalizado.
\end{abstract}

Palavras-chave: Idosos, Instituição de Longa Permanência, Equipe Multiprofissional, Promoção da Saúde.

\section{Health conditions of institutionalized elderly: contributions to interdisciplinary action and health promoter}

\begin{abstract}
Introduction: The evaluation of the health status of the institutionalized elderly is considered an essential practice for the promotion and recovery of health, which should include the investigation of aspects related to biopsychosocial needs. Objective: Clinically characterize the elderly living in a philanthropic ILPI in a city in the interior of the State of Rio Grande do Sul, aiming at the planning of interdisciplinary actions that preserve independence and / or prevent functional dependence of these elderly people. Method: This is a cross-sectional, descriptive and quantitative study, based on the analysis of a document called the Data Sheet that composes the medical records of
\end{abstract}

Autor para correspondência: Rosane Seeger da Silva, Programa de Pós-graduação Distúrbios da Comunicação Humana, Universidade Federal de Santa Maria, Campus I, Prédio 26, Sala 1418, Cidade Universitária, Camobi, CEP 97105-900, Santa Maria, RS, Brasil, e-mail: rosane.seeger@hotmail.com Recebido em Dez. 8, 2017; 1ª Revisão em Mar. 20, 2018; 2a Revisão em Set. 11, 2018; Aceito em Jan. $25,2019$. 
the elderly. The data were: sex, age, ethnicity / race, schooling, previous occupation / occupation, marital status, presence of diseases and medications, as well as maintenance or not of family bond. Results: 67 elderly people, all males, participated in the study. The age ranged from 60 to 105 years, with the majority being in the age group of 70 years or older. It was identified the predominance of white, literate elderly, general and single professionals; visual deficits (corrected majority) and auditory deficits (corrected minority) were more common. The health conditions related to the history of abuse of alcohol and tobacco, systemic and neurodegenerative diseases, their aggravations and unfavorable consequences, as well as excessive use of psychotropic drugs and low level of maintenance of family bonding were highlighted. Conclusion: Biological and psychosocial situations were analyzed, which were addressed by the current team, among others, which require the participation of new health professionals who do not make up the team. These data can help in planning actions aimed at health promotion, including interdisciplinary attention - extended, individualized and collective - to the institutionalized elderly.

Keywords: Elderly, Long-term Institution, Multiprofessional Team, Health Promotion.

\section{Introdução}

O envelhecimento, atualmente, faz parte da realidade da maioria das sociedades, produzindo mudanças no perfil populacional e novos desafios com consequências diretas para o sistema de saúde pública. Trata-se de um processo universal, marcado por mudanças biopsicossociais inerentes ao processo da vida que varia de pessoa para pessoa, de acordo com sua genética, hábitos de vida e meio onde está inserido.

Dados do último censo populacional realizado pelo Instituto Brasileiro de Geografia e Estatística (INSTITUTO..., 2010), revelam um aumento rápido e progressivo da população idosa, gerado pela diminuição das taxas de mortalidade e fecundidade, pelos avanços científicos e tecnológicos (principalmente, na área da saúde), bem como pela implementação de políticas públicas específicas para a referida população (HEIN; ARAGAKI, 2012).

A Política Nacional de Saúde da Pessoa Idosa (PNSPI) destaca que é imprescindível oferecer cuidados sistematizados e adequados ao idoso dependente ou independente, destacando a necessidade de ofertar atenção integral e integrada à saúde, seja em seus lares ou em Instituiçôes de Longa Permanência para Idosos (ILPI) (BRASIL, 2006).

De acordo com a legislação brasileira o cuidado ao idoso dependente é responsabilidade prioritária das famílias; porém tal atribuição pode ser dificultada em função das novas configurações e arranjos familiares, especialmente, pela crescente participação da mulher (tradicional cuidadora) no mercado de trabalho. Tal situaçáo impóe ao Estado e à sociedade em geral a responsabilidade em compartilhar com as famílias o cuidado a esse idoso.

Uma das alternativas de cuidados não familiares são as ILPI (públicas, filantrópicas ou privadas) definidas pela Sociedade Brasileira de Geriatria e Gerontologia (SOCIEDADE..., 2003) como estabelecimentos para atendimento integral de pessoas com 60 anos ou mais, dependentes ou independentes, que não dispóem de condições para permanecer com a família ou em domicílio unicelular. Para a Agência Nacional de Vigilância Sanitária (ANVISA), as ILPI configuram-se como domicílios coletivos de pessoas com idade igual ou superior a 60 anos, com ou sem suporte familiar, em condição de liberdade, dignidade e cidadania (BRASIL, 2005).

Há de ressaltar ainda que desde 2005, têm-se definidas as normas técnicas para o funcionamento das ILPI, por meio da Resoluçáo da Diretoria Colegiada da Agência Nacional de Vigilância Sanitária - RDC/ANVISA no 283, de 26 de setembro de 2005 (BRASIL, 2005). Segundo esta resolução, as ILPI, dentre outras exigências, devem contar com uma equipe multidisciplinar que preste cuidado de acordo com as diversas necessidades de saúde dos residentes (que podem apresentar graus de dependência variável - de um a três - sendo o terceiro o de maior dependência). Tais equipes devem contar, minimamente, com assistentes sociais, profissionais de educação física, enfermeiros e técnicos de enfermagem, médicos, nutricionistas, fisioterapeutas e cuidadores de idosos (BRASIL, 2005; SILVA; SANTOS, 2010). Certamente a participação de outros profissionais como farmacêuticos, fonoaudiólogos, terapeutas ocupacionais, musicoterapeutas, poderia ampliar a atenção à saúde dos idosos institucionalizados o que implica, entre outros aspectos, a manutenção de um bom estado de saúde, para que essa pessoa possa alcançar um máximo de vida ativa e saudável, no ambiente em que está inserida, com autonomia e independência física, psíquica e social (SANTOS et al., 2008).

Nesse sentido, convém conhecer as características da população a qual se assiste; sendo esse conhecimento fundamental para o (re) direcionamento da atenção à medida que potencializa as açóes da equipe 
multiprofissional, sobretudo as de promoção da saúde e prevenção de agravos.

Além do corpo de profissionais, uma ILPI deve contar com infraestrutura adequada à população que atende, instalações e condições adequadas de habitação, vestuário e alimentação, deve promover atividade variadas de lazer, esportivas, culturais, educacionais, proporcionar serviços que atendam a saúde dos idosos e manter o quadro de funcionários com formação específica para o cargo a ser exercido (LENARDT et al., 2006).

A propósito dos conhecimentos anteriormente referidos, da experiência prática e/ou de docência das autoras junto a diferentes ILPI, bem como da necessidade de ampliação de estudos sobre as condiçôes de vida e saúde de idosos institucionalizados e sobre a configuração das equipes profissionais que os atendem, realizou-se o estudo com o objetivo de caracterizar clinicamente os idosos residentes em uma ILPI filantrópica de um município do interior do Rio Grande do Sul (RS), visando o planejamento de açóes interdisciplinares que preservem a independência e/ou previnam dependência funcional de idosos institucionalizados.

\section{Método}

Este estudo caracteriza-se como descritivo, transversal e de abordagem quantitativa (ROUQUAYROL, 2003) realizado a partir de informaçôes indiretas e retrospectivas retiradas dos prontuários dos idosos residentes na referida ILPI que conta com uma equipe de saúde multiprofissional composta por assistente social, cuidadores de idosos, enfermeiro (responsável técnico), técnicos de enfermagem, fisioterapeuta, profissional de educação física, médico, nutricionista e psicólogo. A instituição conta ainda com o apoio de acadêmicos da fisioterapia, fonoaudiologia e terapia ocupacional para complementar a assistência aos idosos e para o desenvolvimento de atividades de ensino, pesquisa e/ou extensão, configurando-se como importante campo de prática das universidades locais.

A ILPI tem capacidade para atender 90 idosos do sexo masculino e por ocasiáo da coleta de dados, contava com 67 idosos. Foram explorados, após autorização institucional, os 67 prontuários (Ficha de cadastros) organizados e mantidos no setor de saúde da ILPI, contando com as seguintes informaçôes: dados de identificação sociodemográfica do idoso; evoluçôes e prescriçôes médicas, da enfermagem, da fisioterapia e da fonoaudiologia, bem como os resultados de exames (laboratoriais e de imagem).

A realização desta pesquisa foi precedida da sua apreciação e aprovação pelo Comitê de Ética em
Pesquisa, conforme o parecer $n^{\circ} 2.048 .595$, em 05/05/2017, ressaltando o cumprimento dos preceitos éticos da autonomia, náo maleficência, beneficência e justiça em pesquisa com seres humanos, conforme consta a Resolução 466/12 do Conselho Nacional de Saúde.

Os dados foram coletados entre os meses de maio a junho de 2017, por duas pesquisadoras. Por meio de um roteiro previamente elaborado, levantaram-se as características sociodemográficas (sexo, idade, etnia/raça, escolaridade, profissão/ocupação anterior à institucionalizaçáo, estado civil e presença de vínculo familiar), clínicas: déficit sensoriais - visão e audição, Hipertensão Arterial Sistêmica (HAS), Diabetes Mellitus (DM), Acidente Vascular Cerebral (AVC), Doença Pulmonar Obstrutiva Crônica (DPOC), Doenças Osteomusculares (DO) e sequelas por fraturas nos últimos seis meses, hábitos nocivos à saúde, bem como os principais grupos de medicamentos utilizados pelos idosos. Tais dados foram tabulados, utilizando-se o programa Microsoft Office Excel 2013, e analisados por meio de estatística descritiva simples. Os resultados foram organizados em tabelas, e discutidos à luz da literatura pertinente e de relevância.

\section{Resultados}

Conforme indicado anteriormente, foram analisados prontuários de 67 idosos da ILPI; a idade variou entre 60 e 105 anos (média de 75,50 \pm 9,99 anos), a maioria $(71,42 \%)$ se encontrava na faixa dos 70 anos ou mais. Quanto à etnia/raça, a maioria $(89,55 \%)$ foi de brancos. Quanto à escolaridade, houve predomínio de alfabetizados $(53,73 \%)$. No que se refere à ocupação, obteve-se que a maioria estava aposentada e desempenhou atividades de serviços gerais. Verificou-se predominância de solteiros (61,19\%). Acrescenta-se que 62,68\% idosos possuíam vínculos familiares (confira Tabela 1).

Apresentam-se na Tabela 2 a distribuição das características clínicas: déficits sensoriais - visão e audição, HAS, DM, AVC, DPOC, DO e sequelas de fraturas nos últimos seis meses. Ressalta-se que os déficits sensoriais - audição e visão - são os únicos claramente indicados nos prontuários.

Quanto aos hábitos nocivos à saúde, destacam-se a história de alcoolismo $(58,20 \%)$ e tabagismo (61,19\%). Identificam-se características clínicas como as doenças neurodegenerativas, seus agravos e consequências desfavoráveis, quais sejam, os distúrbios da comunicação (de expressão, de compreensão verbal, entre outros) e de alimentaçáo, denominados como disfagias (Tabela 3). 
Tabela 1. Distribuição dos idosos institucionalizados, segundo características sociodemográficas, 2017 $(\mathrm{N}=67)$.

\begin{tabular}{|c|c|c|}
\hline Variáveis & $\mathrm{f}$ & $\%$ \\
\hline \multicolumn{3}{|l|}{ Idade } \\
\hline $60-69$ & 18 & 26,86 \\
\hline $70-79$ & 29 & 43,28 \\
\hline $80-89$ & 12 & 17,91 \\
\hline$>90$ & 08 & 11,94 \\
\hline \multicolumn{3}{|l|}{ Etnia/Raça } \\
\hline Branca & 60 & 89,55 \\
\hline Negra & 05 & 7,46 \\
\hline Indígena & 02 & 2,98 \\
\hline \multicolumn{3}{|l|}{ Escolaridade } \\
\hline Alfabetizado & 36 & 53,73 \\
\hline Analfabeto & 31 & 46,26 \\
\hline \multicolumn{3}{|c|}{$\begin{array}{l}\text { Profissão/ocupação anterior à } \\
\text { institucionalização }\end{array}$} \\
\hline Serviços gerais & 18 & 26,86 \\
\hline Não informado & 16 & 23,88 \\
\hline Agricultor & 13 & 19,40 \\
\hline Pedreiro & 03 & 4,47 \\
\hline Professor & 02 & 2,98 \\
\hline Servidor público & 02 & 2,98 \\
\hline Ferroviário & 02 & 2,98 \\
\hline Feirante & 02 & 2,98 \\
\hline Empresário & 02 & 2,98 \\
\hline Motorista & 02 & 2,98 \\
\hline Padre & 01 & 1,49 \\
\hline Pastor & 01 & 1,49 \\
\hline Pintor & 01 & 1,49 \\
\hline Mecânico & 01 & 1,49 \\
\hline Militar & 01 & 1,49 \\
\hline \multicolumn{3}{|l|}{ Aposentadoria } \\
\hline Sim & 64 & 95,52 \\
\hline Não & 03 & 4,47 \\
\hline \multicolumn{3}{|l|}{ Estado civil } \\
\hline Solteiro & 41 & 61,19 \\
\hline Viúvo & 12 & 17,91 \\
\hline Divorciado & 11 & 16,41 \\
\hline Casado & 03 & 4,47 \\
\hline \multicolumn{3}{|l|}{ Vínculo familiar } \\
\hline Possui & 42 & 62,68 \\
\hline Não possui & 25 & 37,31 \\
\hline
\end{tabular}

$\mathrm{N}=$ Número de idosos que participaram da pesquisa; $\mathrm{f}=$ frequência. Fonte: Dados da pesquisa (2017).

Quanto ao grau de dependência foi constatado que 48 idosos $(71,74 \%)$ eram dependentes nas Atividades de Vida Diária (AVD); sendo 32 idosos (47,46\%) frágeis (grau II ou III de dependência), por apresentarem mais de 80 anos ou, então, um ou mais acometimentos de saúde, por exemplo, incontinência urinária ou fecal, queda nos últimos seis meses e dependência para AVD.
Tabela 2. Distribuição das características clínicas: déficits sensoriais: visão e audição e principais doenças entre os idosos institucionalizados, 2017 ( $N=67)$.

\begin{tabular}{llr}
\hline \multicolumn{1}{c}{ Variáveis } & f & $\%$ \\
\hline Acuidade visual & & \\
Déficit visual & 29 & 43,28 \\
Déficit corrigido com órtese & 21 & 31,34 \\
Déficit não corrigido & 08 & 11,94 \\
Sem déficit visual & 13 & 19,40 \\
Não possui registro & 25 & 37,31 \\
Acuidade auditiva & & \\
Déficit auditivo & 20 & 29,85 \\
Déficit corrigido com AASI & 02 & 2,98 \\
Déficit não corrigido & 18 & 26,86 \\
Sem déficit & 22 & 32,83 \\
Não possui registro & 25 & 37,31 \\
Principais doenças & & \\
HAS & 41 & 61,19 \\
DM & 11 & 16,41 \\
AVC & 14 & 20,89 \\
DPOC & 07 & 10,44 \\
DO & 13 & 19,40 \\
Sequelas de Fratura nos & 11 & 16,41 \\
últimos seis meses & & \\
\hline AASI=Apar
\end{tabular}

AASI = Aparelho de Amplificação Sonora Individual; HAS $=$ Hipertensão Arterial Sistêmica; DM $=$ Diabetes Mellitus; $\mathrm{AVC}=$ Acidente Vascular Cerebral; $\mathrm{DPOC}=$ Doença Pulmonar Obstrutiva Crônica; DO = Doenças Osteomusculares; $\mathrm{N}$ = Número de idosos que participaram da pesquisa; $\mathrm{f}=$ frequência. Fonte: Dados da pesquisa (2017).

Tabela 3. Características clínicas dos idosos institucionalizados, $2017(\mathrm{~N}=67)$.

\begin{tabular}{lcr}
\hline \multicolumn{1}{c}{ Variáveis } & f & \multicolumn{1}{c}{$\%$} \\
\hline Processo demencial não & 31 & 46,26 \\
especificado & & \\
Doença de Alzheimer & 08 & 11,94 \\
Doença de Parkinson & 03 & 4,47 \\
Distúrbios da expressão verbal & 37 & 55,22 \\
Distúrbios de compreensão verbal & 25 & 37,31 \\
Disfagias & 11 & 16,41 \\
Incontinência urinária & 29 & 43,28 \\
Incontinência Fecal & 25 & 37,31 \\
\hline
\end{tabular}

$\mathrm{N}=$ Número de idosos que participaram da pesquisa; $\mathrm{f}=$ frequência. Fonte: Dados da pesquisa (2017).

Por fim, destaca-se o consumo de medicamentos pelos idosos da ILPI; apenas um idoso (1,49\%) não fazia uso de medicamentos e $44(71,74 \%)$ estavam submetidos à polifarmácia, ou seja, utilizavam cinco ou mais medicamentos. Na Tabela 4, encontram-se identificados os grupos de medicamentos usados (psicotrópicos, cardiovasculares, metabólicos/gastrointestinais e vitaminas). 
Tabela 4. Principais grupos de medicamentos em idosos institucionalizados, $2017(\mathrm{~N}=67)$.

\begin{tabular}{lcc}
\hline Principais grupos de medicamentos & f & $\%$ \\
\hline Psicotrópicos & 49 & 73,13 \\
Cardiovasculares & 40 & 59,70 \\
Gastrointestinais/metabolismo & 22 & 32,83 \\
Vitaminas & 11 & 16,41 \\
Antiglicemiantes & 08 & 11,94 \\
\hline
\end{tabular}

$\mathrm{N}=$ Número de idosos que participaram da pesquisa; $\mathrm{f}=$ frequência. Fonte: Dados da pesquisa (2017).

\section{Discussão}

O presente estudo permitiu caracterizar as condiçốes de saúde de idosos residentes em uma ILPI filantrópica exclusivamente masculina do interior do RS. Em relação à faixa etária, o predomínio de idosos com idade superior a 70 anos de idade na ILPI, corrobora com a literatura (DEL DUCA et al., 2012; MENEZES et al., 2011). Ainda, as análises deste estudo permitem concluir que as dependências que acompanham a idade avançada contribuíram para a institucionalização dessa população. É importante ressaltar que ao longo do processo de envelhecimento aumentam as situaçóes de dependência. Salienta-se que o declínio físico ou intelectual e maiores chances de apresentarem doenças crônicas não transmissíveis e suas complicaçôes, podem dificultar ainda mais a convivência familiar e aumentar a necessidade de institucionalização (SOUSA et al., 2014).

$\mathrm{O}$ estudo evidenciou que esse grupo etário apresenta uma maior incidência de morbidades e incapacidades (NOGUEIRA et al., 2010). Do nosso ponto de vista, coincidente com os autores, tal fato suscita a necessidade de uma equipe multiprofissional capaz de estabelecer estratégias de promoção à saúde com o intuito de postergar, o máximo possível, o desenvolvimento de doenças ou de agravos das morbidades e, consequentemente, das incapacidades. Quando há pré-existência de morbidades é necessário o desenvolvimento de um plano de cuidados individualizado que possibilita amenizar efeitos dos comprometimentos de saúde e, assim, auxilia os idosos a manterem o autocuidado, de modo a potencializar e preservar a sua autonomia por maior tempo possível.

Ao se analisar a escolaridade e a ocupaçáo dos idosos, verificou-se que existe grande número de idosos não alfabetizados, o que pode ser reflexo das dificuldades de acesso às escolas na época em que eram crianças (poucas escolas e/ou pertencimento ao meio rural), desvalorização da educação formal e, ainda por condiçóes socioeconômicas precárias, características próximas encontradas no estudo de Clares et al. (2011) que investigou as características demográficas, socioeconômicas e de saúde de idosos cadastrados em uma Unidade de Saúde da Família, do município de Fortaleza/CE .

Fagundes et al. (2011), dentre outros autores, discutem que o baixo nível de escolaridade e a idade avançada são fatores de risco para o comprometimento cognitivo e déficit funcional. Enquanto outros (MACHADO; CORREIA; MANSUR, 2007) descrevem que sujeitos mais escolarizados obtêm melhores desempenhos nos testes de linguagem, levando-se em conta as características culturais e acesso à informação. Além disso, o baixo grau de instrução, quando associado às condições econômicas precárias e à dificuldade de acesso à rede de suporte, pode dificultar o autocuidado e o gerenciamento dos problemas de saúde pelo idoso e por sua família, contribuindo para a institucionalização (AIRES; PAZ; PEROSA, 2006).

Outra característica sociodemográfica, encontrada neste estudo e que corrobora outros (PELEGRINI et al., 2008; GUEDES; SILVEIRA, 2004), refere-se à alta porcentagem de solteiros dentre os idosos institucionalizados, o que pode indicar a marginalização do idoso sem família, ou o fato de o próprio idoso solteiro preferir, muitas vezes, a institucionalização, pois acredita ser um incômodo para a família, ou essa, considerá-lo do mesmo modo (GUEDES; SILVEIRA, 2004).

A solidão e a falta de uma pessoa para acolher ou assistir o idoso no âmbito domiciliar, contribuem para o seu encaminhamento e permanência em uma ILPI (FERREIRA et al., 2009). Entretanto, verificou-se, neste estudo, que a maioria dos idosos $(62,68 \%)$ apresentam vínculos familiares, dado diferente do encontrado por Araújo et al. (2008) com idosos institucionalizados, do Distrito Federal, que encontrou percentual de vínculo familiar inferior a $(50,8 \%)$.

Identificou-se que $95,52 \%$ dos idosos desta pesquisa possuíam renda de um salário mínimo proveniente da aposentadoria. Estudo de Pelegrini et al. (2008) também identificou alto índice (100\%) de aposentados com renda de um salário mínimo e, inclusive, indicou que o rendimento dos idosos era integralmente entregue à instituição na forma de pagamento. Tal fato náo ocorre na ILPI deste estudo. Nessa, reserva-se o percentual correspondente a $70 \%$ para a instituição. $\mathrm{O}$ restante pode ficar de posse do próprio idoso, de sua família ou depositado em uma conta bancária do idoso para gastos futuros. Tal situação fortalece o previsto na Lei Orgânica de Assistência Social (LOAS) - lei no 8742/93 
(BRASIL, 1993), na Política Nacional do Idoso (PNI) - lei no 8842/94 (BRASIL, 1994) e citado por Pires e Silva (2001). Segundo os autores a LOAS contempla a organizaçáo das políticas de assistência, dispondo também sobre medidas específicas, como a garantia de um salário mínimo mensal ao idoso que comprovadamente não possuir meios de prover sua própria manutenção ou tê-la provida por sua família.

Quanto às condiçốes de saúde dos idosos, foram encontrados dados semelhantes a outros estudos. Os déficits sensoriais são comuns a idosos institucionalizados, mas há também as alterações temporárias e leves dos processos cognitivos, passando essas, muitas vezes, despercebidas. É necessário distinguir as manifestaçóes iniciais de doença que afetam cognição das naturais modificaçóes associadas ao processo de envelhecimento (ENGELHARDT et al., 2002; MANSUR; VIUDE, 2002; SOUZA; ODA, 2008; CÁCERES, 2012).

No que tange ao sistema visual, pode-se assegurar que ele é um dos primeiros a apresentar as consequências do envelhecimento fisiológico. Entre os 40 e 50 anos inicia-se a presbiopia, a qual se manifesta com redução lenta, contínua e não modificável da capacidade de acomodar ou focalizar objetos próximos, podendo chegar até $80 \%$ de perda quando se aproxima da nona década (MACEDO et al., 2008).

As alteraçôes visuais são tidas como fatores de risco para a dependência funcional de idosos, sendo necessário conhecer tais fatores (RIBEIRO et al., 2015). Este problema pode ser um agravante capaz de produzir, além da dependência, situaçôes de constrangimento para iniciar e manter conversas, dificuldade para sair, isolamento social, perda de papéis ocupacionais, limitaçôes de mobilidade e condiçôes de fragilidade (VEIGA et al., 2016).

Os idosos deste estudo, apresentaram moderado índice de déficit visual (43,28\%), sendo 31,34\% corrigidos com o uso de órtese, fato que ameniza os prejuízos causados pelo déficit. Estes achados corroboram com o estudo desenvolvido por Menezes e Bachion (2012), os quais observaram que $81,4 \%$ da amostra apresentaram alteraçóes visuais, sendo que $44,1 \%$ faziam uso de órteses/dispositivos para correção. No entanto, muitos dos idosos deste estudo (37,31\%) não têm diagnóstico sobre suas condições de visão (situação que precisa ser mais explorada pela equipe de saúde local). A propósito, não foram encontrados estudos que indiquem situação similar a então descrita.

Quanto à audição, também se constatou considerável referência à presença de déficit auditivo (29,85\%), sendo corrigido apenas 2,98\%. Assim como o que ocorre com a condição visual, muitos idosos (37,31\%) não apresentam indicação de perda auditiva, quanto menos diagnóstico (situação que também merece ser melhor abordada pela equipe de saúde). Gazzola et al. (2006), em estudo realizado junto a idosos institucionalizados, encontraram percentual de $75 \%$ de perda auditiva. Note-se discrepância entre os dados deste estudo e o de Gazzola et al. (2006), a qual pode ser explicada pela ausência do profissional de Fonoaudiologia na ILPI. Em estudo realizado com idosos institucionalizados, no município de Santo André/SP, também foi verificada uma frequência superior $(39,3 \%)$ de idosos com perda auditiva diagnosticada à encontrada neste (SOUZA; ODA, 2008).

A presbiacusia é uma das alteraçôes mais incapacitantes do processo de envelhecimento; caracteriza-se por danos do sistema auditivo, os quais resultam na diminuição gradativa da sensibilidade auditiva e na redução da inteligibilidade de fala em níveis supralimiares, comprometendo seriamente o processo de comunicação verbal (KANO; MEZZENA; GUIDA, 2009; MARQUES; KOZLOWSKI; MARQUES, 2004). Portanto, a tendência é de que a perda auditiva na velhice, por ser progressiva, cause prejuízos à qualidade de vida, por exemplo, isolamento, dependência e frustraçóes (CIORBA et al., 2012), sendo, por isso, relevante o implemento de ações dirigidas para diminuir as comorbidades associadas à perda auditiva (MATTOS; VERAS, 2007).

Aspecto importante de ser discutido diz respeito a constataçáo dos agravos de saúde decorrentes da não adoção de hábitos saudáveis ao longo da vida, por exemplo, história de uso abusivo de álcool e de tabaco. Felten et al. (2005) sugeriram que o "alcoolismo" ocorre entre dois e 10\% dos idosos; Ximenes e Côrte (2007) afirmaram que o consumo de álcool, assim como a presença de outras doenças, contribui para a institucionalização de idosos. Sabe-se que o uso abusivo de álcool pode provocar danos cerebrais, mudança estrutural e fisiológica que afetam os processos cognitivos.

Convém destacar, ainda, o alto índice $(61,19 \%)$ de HAS ao lado do DM (16,41\%); tais condiçóes de saúde são resultantes de causas passíveis de prevenção na fase adulta, por meio da adoção de hábitos saudáveis de vida: prática regular de atividades físicas, cessação do tabagismo e do uso de álcool e outras drogas (BRASIL, 2011; COSTA; FEDOSSE; LÈFEVRE, 2014; LOPÉZ- JARAMILLO et al., 2014).

A HAS é um fator de risco grave tanto para as doenças cardiovasculares quanto para os Acidentes Vasculares Cerebrais (AVC); sem dúvidas, a principal 
causa de morte nos últimos tempos (VASCONCELOS, 2002; MALTA et al., 2009). Sabe-se que, após sofrer um AVC, mais da metade das pessoas pode apresentar sequelas que comprometem a qualidade de vida. Segundo Paixão, Silva e Camerini (2010), a fraqueza muscular é a mais prevalente, assim como podem ocorrer distúrbios da comunicação/ linguagem e disfagias.

A propósito do dito acima, foram encontrados idosos com distúrbios de expressão e compreensão verbal $(55,22 \%$ e $37,31 \%$, respectivamente; ressaltando-se que um mesmo sujeito apresentou ambos os distúrbios). Estudo realizado em ILPI, na cidade de Fortaleza/CE, constatou que mais de $50 \%$ dos idosos apresentavam comunicação verbal prejudicada, frequência semelhante à deste estudo (FREITAS; PEREIRA; GUEDES, 2008).

As causas dos distúrbios de comunicação, identificados neste estudo, foram os AVC, os comprometimentos neurológicos de natureza degenerativa diagnosticados (Doença de Parkinson e Doença de Alzheimer) ou não (processos demenciais não identificados). Entre os processos demenciais identificados, o mais frequente foi a doença de Alzheimer (11,94\% dos idosos). Ressalta-se que na busca por diagnósticos, deparou-se com a generalidade (em 46,26\% dos casos, a classificação da demência não estava especificada). Essa dificuldade em identificar a demência é referida por Fornari et al. (2010), que alertam que o diagnóstico das síndromes demenciais é complexo, com fundamentação em largo arcabouço, teórico e prático. Os autores destacam a prática clínica (anamnese e exame físico, testes cognitivos e neuropsicológicos padronizados), os exames de neuroimagem e os laboratoriais. Do nosso ponto de vista, possivelmente, os idosos identificados como em processo demencial (sem especificação) devem-se às dificuldades institucionais para investigação aprofundada das manifestaçôes clínicas desses idosos. Fato que não ocorre quanto ao diagnóstico da Doença de Parkinson (4,47\% dos idosos da ILPI pesquisada). Os sinais deste processo degenerativo (crônico e lentamente progressivo) - rigidez muscular, instabilidade postural, bradicinesia e tremores em repouso - são facilmente observáveis. Este comprometimento acomete um em cada 100 idosos, embora existam casos em que os primeiros sinais aparecem antes de 40 anos (FELTEN et al., 2005). A pessoa com Doença de Parkinson tem dificuldades (ou até perde) a mobilidade e pode sofrer acidentes (quedas) e lesóes na pele (FELTEN et al., 2005).

As DO e suas consequências também foram encontradas neste estudo, corroborando achados sobre tal condição na velhice (PILGER; MENON;
MATHIAS, 2011). Passos e Ferreira (2010), afirmaram que a prevalência de idosos com HAS e DO pode estar relacionada, dentre outros fatores, com estilo de vida sedentário e alimentação inadequada.

Neste estudo, observou-se a presença de DPOC em $10,44 \%$ dos idosos. A DPOC pode ser definida

[...] fisiologicamente como a obstrução crônica ao fluxo aéreo devido a associaçáo de enfisema e obstruçấo das vias aéreas periféricas na bronquite crônica (SENGER, 2006, p. 600)

nos idosos, em geral, a DPOC assume três formas: enfisema, bronquite crônica e asma.

As incontinências - urinária e fecal - afetam 43,28\% e $37,31 \%$ dos idosos deste estudo, respectivamente. Segundo dados epidemiológicos nacionais, a incontinência urinária e fecal aparece em cerca de $30 \%$ das pessoas idosas (institucionalizadas ou não). Muitas das causas são reversíveis visto que podem originar-se em períodos de delírio, restrição de mobilidade, retenção urinária, infecção se ocasionada por efeito de medicamentos. Em muitos casos, tais incontinências estáo relacionadas às repercussóes emocionais e sociais (BRASIL, 2007).

Note-se que muitos idosos deste estudo $(71,74 \%)$ conforme características anteriormente descritas, possuem dependência para atividades do dia a dia e, por isso, podem ser compreendidos como frágeis. A fragilidade é definida como uma síndrome clínica caracterizada por diminuição de reserva e pela resistência reduzida aos estressores, resultante de declínio cumulativo nos sistemas fisiológicos (principalmente neuroendócrino, imunológico e musculoesquelético), causando vulnerabilidade às condiçóes adversas entre os idosos (FRIED et al., 2001, 2004).

Neste contexto, reforça-se a importância da avaliação multidimensional do idoso, como possibilidade de detectar os fatores de risco para fragilidade e atuar de maneira oportuna, buscando evitar o surgimento e/ou o agravo das condiçóes de saúde dos idosos institucionalizados (FABRICIO-WEHBE et al., 2009). Ressalta-se, mais uma vez, que o cuidado aos idosos fragilizados seja realizado por uma equipe multiprofissional, com abordagem interdisciplinar, preparada para identificar e intervir nos problemas de saúde do idoso, objetivando não somente a reabilitação, mas, principalmente, o estabelecimento de medidas preventivas e educativas para a saúde dos idosos institucionalizados (REMOR; BOS; WERLANG, 2011).

Desse modo, pode-se dizer que os achados deste estudo confirmaram aspectos considerados prioritários de intervenção, ainda levantados na década de 1980 
(aumento das doenças crônicas não transmissíveis e das taxas de envelhecimento populacional) os quais impeliram as discussóes relativas à Promoção da Saúde. Nesta perspectiva, alcança-se saúde com autonomia dos sujeitos e dos grupos sociais. Por isso, no Brasil, tem-se uma política específica de Promoção da Saúde, considerada como uma estratégia fundamental para se alcançar a melhoria da qualidade de vida da populaçấo, que requer gestão compartilhada entre usuários, movimentos sociais, trabalhadores do setor sanitário e de outros setores (BRASIL, 2012).

Considerando, pois, o paradigma da Promoção da Saúde, convém que uma equipe multiprofissional e os demais trabalhadores de uma ILPI, conheçam os aspectos característicos do envelhecimento (com e sem comorbidades), que saibam atuar interdisciplinarmente sobre as necessidades dos idosos e que sejam competentes em mobilizar os idosos a buscarem qualidade de vida. Neste sentido, a atuação interdisciplinar apresenta-se como potente arranjo de cuidado em uma ILPI; pode ser alcançada por meio de uma relaçấo de reciprocidade e de mutualidade, exigindo dos profissionais uma compreensão unitária e não fragmentada do fenômeno humano abordado pela equipe. Nos termos de Vilela e Mendes (2003, p. 527), a atuação interdisciplinar requer dos profissionais

[...] flexibilidade, confiança, paciência, intuição, capacidade de adaptação, sensibilidade em relação às demais pessoas, aceitação de riscos, aprender a agir na diversidade, aceitar novos papéis.

Note-se a aproximação dos paradigmas - Promoção da Saúde e Interdisciplinaridade - e a potência dos mesmos para superar alguns aspectos da institucionalização, por exemplo, a realização de poucas atividades físicas, o isolamento, depressão, situaçóes que favorecem o desenvolvimento ou a manutenção de doenças cardiovasculares e de outras que atingem o Sistema Nervoso Central, justificando, assim, a utilização de medicamentos que atuam nesses dois sistemas. Sabe-se também que muitos idosos são institucionalizados pois sofreram maus tratos, abandono e exclusão social, o que predispóe ao desenvolvimento de quadros depressivos e, consequentemente, a utilização de medicamentos (JESUS et al., 2010). A propósito, verificou-se, neste estudo, o consumo de excessiva quantidade de medicamentos pelos idosos $(98,50 \%$ necessitam de medicação de uso contínuo, dos quais $65,67 \%$ fazem uso de cinco ou mais fármacos diferentes, por prescrição médica).

A prática da polifarmácia está associada ao aumento do risco e da gravidade das reaçóes adversas, interaçóes medicamentosas, toxicidade cumulativa, erros de medicação, reduçáo da adesão ao tratamento e elevação da morbimortalidade. Em relação ao tipo de medicamento, os maiores percentuais foram para os psicotrópicos e cardiovasculares. Estudo realizado por Ferreira Junior et al. (2016), com 34 idosos residentes em uma ILPI de um município de Minas Gerais, concluiu que os medicamentos que atuam no sistema cardiovascular eram os mais prescritos, seguidos daquele com ação no Sistema Nervoso Central - SNC. Assim, os dados desta pesquisa corroboram o estudo mineiro.

Conhecer a quantidade e os grupos de medicamentos utilizados pelos idosos, bem como seus efeitos, é fundamental visto que este grupo populacional é propenso ao uso inadequado de drogas e à polifarmácia, elevando, assim, a incidência de interações medicamentosas e reaçóes adversas (NOVAES, 2007). Tais fatos dão maior complexidade ao cuidado cotidiano institucional, ao lado da, também conhecida, perda de papéis dos idosos institucionalizados que interferem negativamente em suas dimensôes social, mental e psíquica.

É importante que os profissionais da equipe multiprofissional, ao compreenderem a multiplicidade e a complexidade dos fatores envolvidos no envelhecimento e na institucionalização, elaborem planos de cuidados e apliquem intervençóes condizentes com as características pessoais, sociais e culturais dos idosos residentes na ILPI. É fundamental reconhecer as especificidades de cada idoso para que se possa favorecer a independência, a autonomia e a continuidade da história de vida/das expectativas de cada um. Do nosso ponto de vista, quando se adotam os paradigmas da Promoção da Saúde e da Interdisciplinaridade, a institucionalização pode ser entendida como um processo, com limitaçóes e desafios, voltados à integraçáo e à melhor qualidade de vida dos idosos que dela necessitem.

Não é recente a discussão sobre a importância da atuaçáo multiprofissional em ILPI, tanto que a resolução RDC/ANVISA no 283 (BRASIL, 2005) preconiza a existência de uma equipe mínima de saúde. No entanto, a Fonoaudiologia não a integra, embora, desde a década de 1990, autores da área (TUBERO et al., 1996; GIACHETI; DUARTE, 1997; BACHA et al., 1999; SILVEIRA; RUSSO, 1999; ALENCAR; PEREIRA; CYSNE, 2003; MOTA et al., 2003; JALES et al., 2005; MENEZES; VICENTE, 2007) revelam sua importância quanto à avaliação, diagnóstico precoce, elaboração de programas educativos, de orientaçóes e de reabilitação das habilidades comunicativas, imprescindíveis para promover uma melhor qualidade de vida dos idosos institucionalizados. Possivelmente, a 
atuação da Fonoaudiologia nas ILPI ainda não é comum devido ao fato de não estar prevista como obrigatória na Resolução no 283/2005 e, ainda, pelo desconhecimento do potencial desta profissão quanto aos múltiplos benefícios relacionados, sobretudo, à independência dos idosos, potencializada pela interação verbal.

Mello, Cáceres e Fedosse (2014) explicam que à medida que o idoso se expressa, ele se (re) organiza (subjetivamente) e se impóe como ser social. Maia, Castro e Jordão (2010) enfatizam que nas ILPI há pouca interação entre os moradores, muito silêncio e muita espera. Diante de tal realidade, destaca-se a importância de a equipe multiprofissional, contar com a participação do fonoaudiólogo, para maximizar o trabalho com a linguagem, como atividade simbólica que ressignifica as histórias heterogêneas de vida de cada sujeito e reorganiza lembranças do passado, experiências do presente e projeta ações no futuro (GAMBURGO; MONTEIRO, 2009; MASSI et al., 2012; MELLO; CÁCERES; FEDOSSE, 2014). Tais autoras referem a linguagem como potente meio de constituição da memória, da subjetividade e da intersubjetividade. De modo que na partilha, na interlocução, o idoso pode se manter ativo e, na ausência dos processos interlocutivos, o idoso institucionalizado tende a se colocar em situação de dependência e, consequentemente, perde autonomia (uma das condições que caracterizam a fragilidade do idoso).

\section{Conclusão}

O presente estudo caracterizou sociodemográfica e clinicamente os idosos residentes em uma ILPI filantrópica de um município do interior do estado do RS. Condiçốes como pouca escolaridade, baixa qualificação profissional e renda, ao lado das condiçôes crônicas de saúde (HAS, DM, DPOC, DO, processos demências, entre outras), foram evidentes, corroborando estudos da área. Ressalta-se que as doenças prevalentes foram as cardiovasculares e as relacionadas aos distúrbios do SNC, necessitando de medicamentos psicotrópicos e cardiovasculares, bem como de processos terapêuticos adequados às necessidades de saúde dos idosos.

Os resultados chamam atenção para a necessidade de cuidados realizados por uma equipe multiprofissional, com abordagem interdisciplinar e assentada no paradigma da Promoção da Saúde, além dos conhecimentos acerca das características do envelhecimento e de cada idoso, visando, assim, um cuidado mais efetivo e eficaz, individualizado/singular, favorecedor de autonomia e de independência do idoso institucionalizado. Idosos institucionalizados e frágeis necessitam de cuidados específicos, que vão do incentivo ao convívio grupal à refinada análise sobre o uso de medicamentos e de recursos assistivos adequados às necessidades de cada um.

Considerando que a população idosa se faz crescente e que as ILPI se configuram na melhor opção para uma parcela de idosos e suas famílias, convém que, cada vez mais, essas possuam infraestrutura e recursos humanos competentes para bem atender essa clientela, conhecendo as especificidades relacionadas às diferentes faixas etárias e, buscando a manutençâo da independência funcional e da qualidade de vida dos idosos institucionalizados.

Destaca-se que além da análise contínua das condiçóes de saúde dos idosos, é imprescindível a análise da estrutura e do funcionamento institucional sob os pontos de vista dos diferentes sujeitos implicados, de modo que possa melhorar a assistência ofertada no âmbito das ILPI.

Por fim, como limitações do estudo pôde-se apontar o próprio delineamento da pesquisa, pois o fato de ser um estudo transversal impossibilita o estabelecimento das relaçóes causais entre as variáveis estudadas. Sugere-se que estudos futuros sejam realizados com delineamento longitudinal e acompanhamento com visão ampliada das condiçôes de saúde e dos aspectos fonoaudiológicos de idosos institucionalizados, como forma de subsidiar açóes de identificação e intervenção precoces, bem como complementar esses achados.

\section{Referências}

AIRES, M.; PAZ, A. A.; PEROSA, C. T. O grau de dependência e características de pessoas idosas institucionalizadas. Revista Brasileira de Ciências do Envelhecimento Humano, Passo Fundo, v. 3, n. 2, p. 79-91, 2006.

ALENCAR, S. C.; PEREIRA, S. M. S.; CYSNE, C. O processo de envelhecimento e as características da função mastigatória. Revista CEFAC, São Paulo, v. 3, n. 5, p. $247-$ 250, 2003.

ARAÚJO, N. P. et al. Aspectos sociodemográficos, de saúde e nível de satisfaçáo de idosos institucionalizados no Distrito Federal. Revista de Ciências Médicas, Campinas, v. 17, n. 3-6, p. 123-132, 2008.

BACHA, S. M. C. et al. Perfil fonoaudiológico do idoso institucionalizado. Pró-Fono, São Paulo, v. 11, n. 2, p. $1-7,1999$

BRASIL. Lei n ${ }^{\circ} 8.742$, de 7 de dezembro de 1993. Dispóe sobre a organizaçáo da assistência social e dá outras providências, e legislação correlata. Diário Oficial [da] República Federativa do Brasil, Poder Executivo, Brasília, DF, 7 dez. 1993. 
BRASIL. Lei ${ }^{\circ}$ 8.842, de 4 de janeiro de 1994. Dispóe sobre a Política Nacional do Idoso, cria o Conselho Nacional do Idoso e dá outras providências. Diário Oficial [da] República Federativa do Brasil, Poder Executivo, Brasília, DF, 4 jan. 1994.

BRASIL. Resolução de Diretoria Colegiada - RDC n ${ }^{\circ} 283$, de 26 de setembro de 2005. Regulamento técnico para o funcionamento de instituiçóes de longa permanência para idosos. Diário Oficial [da] República Federativa do Brasil, Poder Executivo, Brasília, DF, 26 set. 2005.

BRASIL. Portaria n ${ }^{\circ} 2528$, de 10 de outubro de 2006. Dispóe sobre a Política Nacional da pessoa idosa. Diário Oficial [da] República Federativa do Brasil, Poder Executivo, Brasília, DF, 19 out. 2006. Disponível em: <http://bvsms. saude.gov.br/bvs/saudelegis/gm/2006/prt2528_19_10_2006. html>. Acesso em: 10 nov. 2017.

BRASIL. Ministério da Saúde. Secretaria de Vigilância em Saúde. Departamento de Vigilância Epidemiológica. Informe técnico: campanha nacional de vacinação do idoso. Brasília, 2007. Disponível em: <http://portal.saude.gov.br/ portal/arquivos/pdf/informe_tecnico_vacina_2007_idoso. pdf>. Acesso em: 13 dez. 2011.

BRASIL. Ministério da Saúde. Secretaria de Vigilância em Saúde. Departamento de Análise de Situação de Saúde. Plano de açōes estratégicas para o enfrentamento das doenças crônicas não transmissiveis (DCNT) no Brasil 2011-2022. Brasília, 2011.

BRASIL. Ministério da Saúde. Glossário temático: promoção da saúde. Brasília, 2012.

CÁCERES, J. V. Aspectos fonoaudiológicos e qualidade de vida de idosos residentes em um municipio de pequeno porte do RS. 2012. 165 f. Dissertação (Mestrado em Distúrbios da Comunicação Humana) - Universidade Federal de Santa Maria, Santa Maria, 2012.

CIORBA, A. et al. The impact of hearing loss on the quality of life of elderly adults. Clinical Interventions in Aging, New Zealand, v. 7, p. 159-163, 2012. http://dx.doi. org/10.2147/CIA.S26059.

CLARES, J. W. B. et al. Perfil de idosos cadastrados numa Unidade Básica de Saúde da Família de Fortaleza/CE. Revista da Rede de Enfermagem do Nordeste, Fortaleza, v. 12, p. 988-994, 2011. Número Especial.

COSTA, M. L. G.; FEDOSSE, E.; LEFÈVRE, A. P. Doenças crônicas não transmissíveis: cuidado em Fonoaudiologia. In: MARCHESAN, I. Q.; SILVA, H. J.; TOMÉ, M. C. Tratado das especialidades em Fonoaudiologia. Rio de Janeiro: Guanabara Koogan, 2014. p. 806-813.

DEL DUCA, G. F. et al. Indicadores da institucionalização de idosos: estudo de casos e controles. Revista de Saúde Pública, São Paulo, v. 46, n. 1, p. 147-153, 2012. http:// dx.doi.org/10.1590/S0034-89102012000100018.

ENGELHARDT, E. et al. Idosos institucionalizados: rastreamento cognitivo. Revista de Psiquiatría Clínica, Maringá, v. 25, n. 2, p. 74-79, 2002.
FABRÍCIO-WEHBE, S. C. C. et al. Crosscultural adaptation and validity of the Edmonton Frail Scale-EFS in a brazilian elderly sample. Revista Latino-Americana de Enfermagem, Ribeirão Preto, v. 17, n. 6, p. 1043-1049, 2009. http://dx.doi.org/10.1590/S0104-11692009000600018.

FAGUNDES, S. D. et al. Prevalence of dementia among elderly Brazilians: a systematic review. Sao Paulo Medical Journal, São Paulo, v. 129, n. 1, p. 46-50, 2011. http:// dx.doi.org/10.1590/S1516-31802011000100009.

FELTEN, B. S. et al. Geriatria e gerontologia. Rio de Janeiro: Reichmann e Autores Editores, 2005.

FERREIRA JUNIOR, C. L. et al. Análise das interaçóes medicamentosas em prescriçóes de uma Instituição de Longa Permanência em um Munícipio de Minas Gerais. Boletim Informativo Geum, Teresina, v. 7, n. 1, p. 64-70, 2016.

FERREIRA, R. C. et al. Saúde bucal de idosos residentes em instituiçôes de longa permanência de Belo Horizonte, Minas Gerais, Brasil. Cadernos de Saúde Pública, Rio de Janeiro, v. 25, n. 11, p. 2375-2385, 2009.

FORNARI, L. H. T. et al. As diversas faces da síndrome demencial: como diagnosticar clinicamente? Scientia Medica, Porto Alegre, v. 20, n. 2, p. 185-193, 2010.

FREITAS, M. C.; PEREIRA, R. F.; GUEDES, M. V. C. Diagnósticos de enfermagem em idosos dependentes residentes em uma instituição de longa permanência em Fortaleza/CE. Ciências, Cuidado e Saúde, Maringá, v. 9, n. 3, p. 518-526, 2008.

FRIED, L. P. et al. Frailty in older adults: evidence for a phenotype. The Journals of Gerontology: Series A, Biological Sciences and Medical Sciences, Washington, v. 56, n. 3, p. 146-156, 2001.

FRIED, L. P. et al. Untangling the concepts of disability, frailty and comorbidity: implications for improved targeting and a care. The Journals of Gerontology: Series A, Biological Sciences and Medical Sciences, Washington, v. 59, n. 3, p. 255-263, 2004. PMid:15031310.

GAMBURGO, L. J. L.; MONTEIRO, M. I. B. Singularidades do envelhecimento: reflexôes com base em conversas com um idoso institucionalizado. Interface: Comunicação, Saúde, Educação, Botucatu, v. 13, n. 28, p. 31-41, 2009.

GAZZOLA, J. M. et al. Caracterização clínica de idosos com disfunção vestibular crônica. Revista Brasileira de Otorrinolaringologia, São Paulo, v. 72, n. 4, p. 1-13, 2006.

GIACHETI, C. M.; DUARTE, V. G. Programa de atuação fonoaudiológica junto a idosos institucionalizados. In: LAGROTTA, M. G. M.; CÉSAR, C. P. H. A. A fonoaudiologia nas instituiçôes. São Paulo: Lovise, 1997. p. 17-27.

GUEDES, J. M.; SILVEIRA, R. C. R. Análise da capacidade funcional da população geriátrica institucionalizada na cidade de Passo Fundo, RS. Revista Brasileira de Ciências do Envelhecimento Humano, Passo Fundo, v. 1, n. 2, p. 10-21, 2004. 
HEIN, M. A.; ARAGAKI, S. S. Saúde e envelhecimento: um estudo de dissertaçóes de mestrado brasileiras (20002009). Ciência \& Saúde Coletiva, Rio de Janeiro, v. 17, n. 8, p. 2141-2150, 2012.

INSTITUTO BRASILEIRO DE GEOGRAFIA E ESTATÍSTICA - IBGE. Sintese de indicadores sociais: uma análise das condiçôes de vida da população brasileira. Rio de Janeiro, 2010. Disponível em: <https://www.ibge.gov. br/home/presidencia/noticias/imprensa/ppts/0000000144. pdf>. Acesso em: 10 out. 2017.

JALES, M. A. et al. Características do sistema estomatognático em idosos: diferenças entre instituição pública e privada. Revista CEFAC, Campinas, v. 7, n. 2, p. 178-187, 2005.

JESUS, I. S. et al. Cuidado sistematizado a idosos com afeccão demencial residentes em instituição de longa permanência. Revista Gaúcha de Enfermagem, Porto Alegre, v. 31, n. 2, p. 285-292, 2010. http://dx.doi.org/10.1590/ S1983-14472010000200012.

KANO, C. E.; MEZZENA, L. H.; GUIDA, H. L. Estudo comparativo da classificação do grau de perda auditiva em idosos institucionalizados. Revista CEFAC, Campinas, v. 11, n. 3, p. 473-477, 2009. http://dx.doi.org/10.1590/ S1516-18462009005000024.

LENARDT, M. H. et al. O idoso institucionalizado e a cultura de cuidadores profissionais. Cogitare Enfermagem, Curitiba, v. 11, n. 2, p. 117-123, 2006.

LOPÉZ-JARAMILLO, P. et al. Consenso latino-americano de hipertensão em pacientes com diabetes tipo 2 e síndrome metabólica. Arquivos Brasileiros de Endocrinologia \& Metabologia, São Paulo, v. 58, n. 3, p. 205-225, 2014.

MACEDO, B. G. et al. Impacto das alteraçôes visuais nas quedas, desempenho funcional, controle postural e no equilíbrio dos idosos: uma revisão de literatura. Revista Brasileira de Geriatria e Gerontologia, Rio de Janeiro, v. 11, n. 3, p. 419-432, 2008. http://dx.doi.org/10.1590/18099823.2008.110310.

MACHADO, O.; CORREIA, S. M.; MANSUR, L. L. Desempenho de adultos brasileiros normais na prova semântica: efeito da escolaridade. Pró-Fono, São Paulo, v. 19, n. 3, p. 289-294, 2007. http://dx.doi.org/10.1590/ S0104-56872007000300007.

MAIA, G. F.; CASTRO, G. D.; JORDÃO, A. B. Ampliando a clínica com idosos institucionalizados. Revista Mal-Estar e Subjetividade, Fortaleza, v. 10, n. 1, p. 193-210, 2010.

MALTA, D. C. et al. Doenças crônicas não-transmissiveis: mortalidade e fatores de risco no Brasil, 1990 a 2006. Brasília: Ministério da Saúde, 2009.

MANSUR, L. L.; VIUDE, A. Aspectos fonoaudiológicos do envelhecimento. In: NETTO, M. P. Gerontologia: a velhice e envelhecimento em visão globalizada. São Paulo: Atheneu, 2002. p. 284-296.

MARQUES, A. C. O.; KOZLOWSKI, L.; MARQUES, J. M. Reabilitação auditiva no idoso. Revista Brasileira de
Otorrinolaringologia, São Paulo, v. 70, n. 6, p. 806-811, 2004. http://dx.doi.org/10.1590/S0034-72992004000600017.

MASSI, G. et al. Práticas intergeracionais e linguageiras no processo de envelhecimento ativo. In: BERBERIAN, A. P.; SANTANA, A. P. Fonoaudiologia em contextos grupais: referenciais teóricos e práticos. São Paulo: Plexus, 2012. p. 33-59.

MATTOS, L. C.; VERAS, R. P. A prevalência da perda auditiva em uma população de idosos da cidade do Rio de Janeiro: um estudo seccional. Revista Brasileira de Otorrinolaringologia, São Paulo, v. 73, n. 5, p. 654-659, 2007.

MELlO, J. G.; CÁCERES, J. V.; FEDOSSE, E. Os processos de negociação de sentido em narrativas orais de idosos. Distúrbios da Comunicação, São Paulo, v. 26, n. 1, p. 131-143, 2014.

MENEZES, L. N.; VICENTE, L. C. C. Envelhecimento vocal em idosos institucionalizados. Revista CEFAC, Campinas, v. 9, n. 1, p. 90-98, 2007. http://dx.doi. org/10.1590/S1516-18462007000100012.

MENEZES, R. L. et al. Estudo longitudinal dos aspectos multidimensionais da saúde de idosos institucionalizados. Revista Brasileira de Geriatria e Gerontologia, Rio de Janeiro, v. 14, n. 3, p. 485-496, 2011.

MENEZES, R. L.; BACHION, M. M. Condições visuais autorrelatadas e quedas em idosos institucionalizados. Revista Brasileira de Oftalmologia, Rio de Janeiro, v. 71, n. 1, p. 23-27, 2012.

MOTA, H. B. et al. Triagem vocal em idosas institucionalizadas. Revista da Sociedade Brasileira de Fonoaudiologia, São Paulo, v. 8, n. 2, p. 58-63, 2003

NOGUEIRA, S. L. et al. Fatores determinantes da capacidade funcional em idosos longevos. Brazilian Journal of Physical Therapy, Sáo Carlos, v. 14, n. 4, p. 322-329, 2010. http:// dx.doi.org/10.1590/S1413-35552010005000019.

NOVAES, M. R. C. G. Assistência farmacêutica ao idoso: uma abordagem multiprofissional. Brasília: Thesaurus, 2007.

PAIXÃO, C. T.; SILVA, L. D.; CAMERINI, F. G. Perfil da disfagia após um acidente vascular cerebral: uma revisão integrativa. Revista da Rede de Enfermagem do Nordeste, Fortaleza, v. 11, n. 1, p. 181-190, 2010.

PASSOS, J. P.; FERREIRA, K. S. Caracterização de uma instituição de longa permanência para idosos e avaliação da qualidade nutricional da dieta oferecida. Alimentos e Nutrição, Araraquara, v. 21, n. 2, p. 241-249, 2010. Disponível em: <http://200.145.71.150/seer/index.php/ alimentos/article/view/1027/a10v21n2.pdf>. Acesso em: 15 out. 2017.

PELEGRINI, A. K. A. P. et al. Idosos de uma instituição de longa permanência de Ribeirão Preto: níveis de capacidade funcional. Arquivos de Ciências da Saúde, São José do Rio Preto, v. 15, n. 4, p. 182-188, 2008.

PILGER, C.; MENON, M. H.; MATHIAS, T. A. F. Características sociodemográfica e de saúde de idosos: contribuiçóes para os serviços de saúde. Revista Latino 
Americana de Enfermagem, Ribeirão Preto, v. 19, n. 5, p. 1230-1238, 2011. http://dx.doi.org/10.1590/S010411692011000500022 .

PIRES, Z. R. S.; SILVA, M. J. Autonomia e capacidade decisória dos idosos de baixa renda: uma problemática na saúde do idoso. Revista Eletrônica de Enfermagem, Goiânia, v. 3, n. 2, [s.p.], 2001. Disponível em: <https://www.fen. ufg.br/revista/revista3_2/autonomia.html>. Acesso em: 12 out. 2017.

REMOR, C. B.; BÓS, A. J. G.; WERLANG, M. C. Características relacionadas ao perfil de fragilidade no idoso. Scientia Medica, Porto Alegre, v. 21, n. 3, p. 107112, 2011.

RIBEIRO, D. K. M. N. et al. Fatores contributivos para a independência funcional de idosos longevos. Revista da Escola de Enfermagem da USP, São Paulo, v. 49, n. 1, p. 89-95, 2015. http://dx.doi.org/10.1590/S0080623420150000100012.

ROUQUAYROL, M. Z. Epidemiologia \& saúde. Rio de Janeiro: Medsi, 2003.

SANTOS, S. S. C. et al. The nurse role in the seniors' long permanence institution. Revista de Enfermagem UFPE, Recife, v. 2, n. 3, p. 262-268, 2008. http://dx.doi. org/10.5205/0203200812.

SENGER, J. Doença pulmonar obstrutiva crônica. In: FREITAS, E. V. et al. Tratado de Geriatria e Gerontologia. Rio de Janeiro: Guanabara Koogan, 2006. p. 600-609.

SILVA, B. T.; SANTOS, S. S. C. Cuidados aos idosos institucionalizados: opinióes do sujeito coletivo enfermeiro para 2026. Acta Paulista de Enfermagem, São Paulo, v. 23, n. 6, p. 775-781, 2010.

SILVEIRA, K. M. M.; RUSSO, I. C. P. A percepção da deficiência auditiva em um grupo de idosos institucionalizados da cidade de Franca. Arquivos de Geriatria e Gerontologia, Rio de Janeiro, v. 3, n. 2, p. 45-51, 1999.
SOCIEDADE BRASILEIRA DE GERIATRIA E GERONTOLOGIA - SBGG. Instituição de longa permanência para idosos: manual de funcionamento. São Paulo, 2003.

SOUZA, A.; ODA, A. L. A importância do levantamento de queixas de idosos institucionalizados durante a entrevista para o planejamento da reabilitaçáo fonoaudiológica. $O$ Mundo da Saúde, São Paulo, v. 32, n. 2, p. 157-169, 2008.

SOUSA, K. T. et al. Baixo peso e dependência funcional em idosos institucionalizados de Uberlândia (MG), Brasil. Ciência \& Saúde Coletiva, Rio de Janeiro, v. 19, n. 8, p. 3513-3520, 2014.

TUBERO, A. L. et al. A linguagem do envelhecer entre muros. In: MARCHESAN, I. Q.; ZORZI, J. L.; GOMES, I. C. D. (Ed.). Tópicos em fonoaudiologia. São Paulo: Lovise, 1996. p. 215-235.

VASCONCELOS, A. M. N. Causas de morte em idosos no Brasil. In: ENCONTRO NACIONAL DE ESTUDOS POPULACIONAIS, 14.; ENCONTRO DA ASSOCIAÇÃO BRASILEIRA DE ESTUDOS POPULACIONAIS, 13., 2002, Ouro Preto. Anais... São Paulo: ABEP, 2002.

VEIGA, B. et al. Avaliaçáo de funcionalidade e incapacidade de idosos longevos em acompanhamento ambulatorial utilizando a WHODAS 2.0. Revista Brasileira de Geriatria e Gerontologia, Rio de Janeiro, v. 19, n. 6, p. 1015-1021, 2016.

VILELA, E. M.; MENDES, I. J. M. Interdisciplinaridade e saúde: estudo bibliográfico. Revista Latino-Americana de Enfermagem, Ribeirão Preto, v. 11, n. 4, p. 525-531, 2003. http://dx.doi.org/10.1590/S0104-11692003000400016.

XIMENES, M. B.; CÔRTE, B. B. A instituição asilar e seus fazeres cotidianos: um estudo de caso. Estudos Interdisciplinares do Envelhecimento, Porto Alegre, v. 11, n. 1, p. 29-52, 2007.

\section{Contribuição dos Autores}

Rosane Seeger da Silva foi responsável pela concepção, redação e organização do texto e de fontes. Elenir Fedosse foi responsável pela orientação em todas as fases e pela revisão final do texto. Fernanda dos Santos Pascotini foi responsável organização do texto. Estefânia Brunelli Riehs foi responsável pela tradução para a língua inglesa. Todos os autores aprovaram a versão final do texto. 